\title{
A New Method of Central Difference INTERPOLATION
}

\author{
Md. Jashim Uddin ${ }^{1}$, Md. Kowsher ${ }^{2}$ And Mir Md. Moheuddin ${ }^{3}$ \\ 1,2,3 Dept. of Applied Mathematics, Noakhali Science and Technology University, \\ Noakhali-3814, Bangladesh
}

\begin{abstract}
In Numerical analysis, interpolation is a manner of calculating the unknown values of a function for any conferred value of argument within the limit of the arguments. It provides basically a concept of estimating unknown data with the aid of relating acquainted data. The main goal of this research is to constitute a central difference interpolation method which is derived from the combination of Gauss's third formula, Gauss's Backward formula and Gauss's forward formula. We have also demonstrated the graphical presentations as well as comparison through all the existing interpolation formulas with our propound method of central difference interpolation. By the comparison and graphical presentation, the new method gives the best result with the lowest error from another existing interpolationformula.
\end{abstract}

\section{KEYWORDS}

Central Difference, Interpolation, Gauss's Formula.

\section{INTRODUCTION}

Interpolation, which is a process of enumerating intermediate value of a function from a set of given or tabular values of that function and is defined as 'the art of reading between the lines of a table \{Hummel (1947), Erdos \& Turan(1938) et al $\}$. The technique of achieving the function's value outside the given limit of arguments is acquainted as extrapolation. Besides, the term interpolation is used for both schemes. Interpolation is a numerical system of calculating the unknown dependent variable's value by means of independent variables and extensively applied in several sorts of fields such as statistics, applied mathematics, data predicting, economics as well as business problems. In practical life, the term interpolation is usually a tool for generating the data analogous and the term extrapolation is always used to find out the future's values, even forecasting future data. Interpolation can assistance to count the economic magnificence like unemployment problems, population and price growth problems etc. Interpolation is widely engaged in significant tool for science, engineering and medical exploration etc. The derivation of computational manner for numerical integration, solutions, differentiations are the most useful applications of interpolation.

Further, there are lots of interpolation methods, for instance, Newton's forward and backward interpolation formula, Newton's divided difference formula, Lagrange's interpolation formula, Central difference interpolation formula, for example, Gauss's formula, Starling's formula, Bessel's formula and Laplace Everett's formula which are attainable in the literature's view of numerical analysis \{Bathe \& Wilson (1976), Jan (1930), Hummel (1947) et al\}. The formula of interpolation with equal intervals are Newton's Gregory forward and backward interpolation. The formula of interpolation with unequal intervals are Newton's general interpolation formula and Lagrange's interpolation formula.

DOI : 10.5121/mathsj.2019.6301 
What is more, the Lagrange interpolation method is a renowned Classical technic for interpolation as well as exhausted to use in numerical problems. Newton's forward and backward methods, that are best appropriated for interpolating near the beginning and end of tabulated values respectively. In addition, these are unusable to interpolate near the central value of a difference table. For interpolation or gaining more proper results near the middle of the table, central difference interpolation methods are most preferable

What is more, the Lagrange interpolation method is a renowned Classical technic for interpolation as well as exhausted to use in numerical problems. Newton's forward and backward methods, that are best appropriated for interpolating near the beginning and end of tabulated values respectively. In addition, these are unusable to interpolate near the central value of a difference table. For interpolation or gaining more proper results near the middle of the table, central difference interpolation methods are most preferable.

Mathematically, suppose the function $\mathrm{y}=\mathrm{f}(\mathrm{x})$ be the functional relation involving variable $\mathrm{x}$ and $\mathrm{y}$. If $\mathrm{x}$ receives the values $\mathrm{x}_{0}-2 \mathrm{~h}, \mathrm{x}_{0}-\mathrm{h}, \mathrm{x}_{0}, \mathrm{x}_{0}+2 \mathrm{~h}$ and the corresponding values of $\mathrm{y}$ which are $\quad \mathrm{y}_{-2,} \mathrm{y}_{-1}, \mathrm{y}_{1}$ and $\mathrm{y}_{2}$.Then we can get the difference table in the two notations $\ldots \ldots$ as follows applying the $\Delta=\delta E^{1 / 2}$, i.e. $\delta=\Delta E^{1 / 2}$, acquainted as central difference table.

\begin{tabular}{|c|c|c|c|c|c|}
\hline $\mathrm{x}$ & $\mathrm{y}$ & $\begin{array}{l}\text { First } \\
\text { difference }\end{array}$ & $\begin{array}{l}\text { Second } \\
\text { difference }\end{array}$ & $\begin{array}{l}\text { Third } \\
\text { difference }\end{array}$ & $\begin{array}{l}\text { Fourth } \\
\text { difference }\end{array}$ \\
\hline$x_{o}-2 h$ & $y_{-2}$ & $\Delta y_{-2}$ & & & \\
\hline$x_{o}-h$ & $y_{-1}$ & $\Delta y_{-1}$ & $\Delta^{2} y_{-2}$ & $\Delta^{3} y_{-2}$ & \\
\hline$x_{o}$ & $y_{0}$ & $\Delta y_{0}$ & $\Delta^{2} y_{-1}$ & $\Delta^{3} y_{-1}$ & $\Delta^{4} y_{-2}$ \\
\hline $\begin{array}{l}x_{o}+h \\
x_{o}+h\end{array}$ & $\begin{array}{l}y_{1} \\
y_{2}\end{array}$ & $\Delta y_{1}$ & $\Delta^{2} y_{0}$ & & \\
\hline
\end{tabular}

To acquire function's values near the middle of a table where central difference interpolation formulas are applicable in which our new method is very suitable. This method employs differences lying as nearly as possible on a horizontal line through $\mathrm{y}_{0}$ in a diagonal difference table.

In our paper, we have established a central difference interpolation formula which is derived from Gauss's third formula and another formula, then we get the new formula or a new method of central difference interpolation.

\section{RELATIVE WORKS}

In the area of numerical analysis, interpolation is a statistical manner to facilitate the complicated functions by modeling the known values as well as interpolating them using an easier function, exerted to estimate the unknown values or price of goods. Due to the simpler way of polynomials, polynomials are generally applied for interpolation, even really assistant to evaluate, differentiate and integrate, called polynomial interpolation. 
Baak, M. et al. [1] present a prescription for the interpolation between multi-dimensional distribution templates based on one or multiple model parameters and the technique utilizes a linear combination of templates, each generated applying fixed values of the model's parameters and transformed according to a specific procedure, to model a non-linear dependency on model parameters. In [2], Bhunia et al.

Applied a portable global positioning system (GPS) in order to collect coordinates of each sample site. Nevertheless, they had used five interpolation methods such as inverse distance weighting (IDW), local polynomial interpolation (LPI), radial basis function (RBF), ordinary kriging (OK) and Empirical Bayes kriging (EBK) for the purpose of generating a spatial distribution of SOC. Gentilucci et al. [3], have proposed an innovative way to interpret data linking precipitation to many topographic parameters and their new model also highlights the presence of some outliers in the rainfall values, which may lead to a better comprehension of climatic dynamics in their area. In [4], Zhang et al. have presented a numerical scheme for solving the non-dissipative Degasperis-Procesi equation based on the u-p formulation. In their paper, the cubic B-spline quasi-interpolation coupled with the finite difference method is used to approximate the spatial derivatives and an optimal third order TVD Runge-Kutta method to calculate the time derivative of the dependent variable. Su, Y. et al. [5] propose a thorough analysis of interpolation bias for the IC-GN algorithm. In their approach, a theoretical model was made to analytically characterize the dependence of interpolation bias upon speckle image, target image interpolation, and reference image gradient estimation. In [6], Dehghan et al. investigate a new meshless numerical technique which is proposed for solving Green-Naghdi equation by combining the moving Kriging interpolation shape functions with the weighted essentially non-oscillatory (WENO) method and their proposed method is based on the nonpolynomial WENO procedure with a view to increasing the convergence order and local accuracy. Besides, in their paper, four examples have been solved that they have shown the efficiency and accuracy of the proposed method.

However, in [7], Li, J. et al. compared the performance of 23 methods, including RF, support vector machine (SVM), ordinary kriging (OK), inverse distance squared (IDS), and their combinations (i.e., RFOK, RFIDS, SVMOK and SVMIDS), using mud content samples in the southwest Australian margin. They also tested the sensitivity of the combined methods to input variables and the accuracy of averaging predictions of the most accurate methods. Bui, T. Q. et al. [8], propose a novel truly meshfree method in which the displacement field is approximated by the radial point interpolation method (RPIM) regardless of predefined mesh, and the domain integrals are evaluated by the so-called Cartesian transformation method (CTM) to obviate the need for a background cell as well as they demonstrated the accuracy and the efficiency of the present formulation by examining a series of numerical examples. In [9], Ghehsareh, et al.

Formulate an efficient numerical technique to solve two-dimensional time fractional cable equation and The performance and accuracy of the method are studied and verified through numerical experiments. Moreover, in their paper, the convergence rate of the temporal discretization scheme is investigated numerically. In [10], Jung, K. H. has done their investigation on the embedding capacity and the visual image quality for the sake of analyzing and recounting reversible data hiding methods using interpolation techniques.

Apart from this works, we propounded a new method of central difference interpolation formula for obtaining the best accuracy among existing methods and the rate of convergence of this new method is almost high. As a result, our new method is extremely effective, even very close to the exact value for the several problems. That's why we have succeeded through our new method to get fewer error values as well as finally compared this with other existing methods. 
Applied Mathematics and Sciences: An International Journal (MathSJ), Vol. 6, No. 2/3, September 2019

\section{Materials And Methods}

Existing formulas and the new method of central difference interpolation: The CentralDifference interpolation formulas are given below

\subsection{Gauss's Forward Formula}

$$
\mathrm{y}(\mathrm{x})=\mathrm{y}_{0}+\mathrm{u} \Delta \mathrm{y}_{0}+\frac{\mathrm{u}(\mathrm{u}-1)}{2 !} \Delta^{2} \mathrm{y}_{-1}+\frac{\mathrm{u}\left(\mathrm{u}^{2}-1\right)}{3 !} \Delta^{3} \mathrm{y}_{-1}+\frac{\mathrm{u}\left(\mathrm{u}^{2}-1\right)(\mathrm{u}-2)}{4 !} \Delta^{4} \mathrm{y}_{-2}+\ldots \ldots
$$

\subsection{Gauss's Backward Formula}

$$
\mathrm{y}(\mathrm{x})=\mathrm{y}_{0}+\mathrm{u} \Delta \mathrm{y}_{-1}+\frac{\mathrm{u}(\mathrm{u}+1)}{2 !} \Delta^{2} \mathrm{y}_{-1}+\frac{\mathrm{u}\left(\mathrm{u}^{2}-1\right)}{3 !} \Delta^{3} \mathrm{y}_{-2}+\frac{\mathrm{u}\left(\mathrm{u}^{2}-1\right)(\mathrm{u}+2)}{4 !} \Delta^{4} \mathrm{y}_{\cdot 2}+
$$

\subsection{Third Gauss's formula}

To derive the Third Gauss's formula, we advance the subscripts in Gauss's Backward Formula by one unit and replacing $\mathrm{u}$ by $\mathrm{u}-1$ then we obtain,

$$
\begin{aligned}
& \mathrm{y}(\mathrm{x})=\mathrm{y}_{1}+(\mathrm{u}-1) \Delta \mathrm{y}_{0}+\frac{\mathrm{u}(\mathrm{u}-1)}{2 !} \Delta^{2} \mathrm{y}_{0}+\frac{\mathrm{u}(\mathrm{u}-1)(\mathrm{u}-2)}{3 !} \Delta^{3} \mathrm{y}_{-1}+\frac{\mathrm{u}\left(\mathrm{u}^{2}-1\right)(\mathrm{u}-2)}{4 !} \Delta^{4} \mathrm{y}_{-1}+ \\
& \frac{\mathrm{u}\left(\mathrm{u}^{2}-1\right)(\mathrm{u}-2)(\mathrm{u}-3)}{5 !} \Delta^{5} \mathrm{y}_{-2}+\ldots \ldots
\end{aligned}
$$

\subsection{Stirling's Interpolation Formula}

Taking the Mean of the Gauss's Forward formula and the Gauss's Backward formula we get Stirling's interpolation formula as

$$
\begin{aligned}
& \mathrm{y}(\mathrm{x})=\mathrm{y}_{0}+\frac{\mathrm{u}\left(\Delta \mathrm{y}_{-1}+\Delta \mathrm{y}_{0}\right)}{2}+\frac{\mathrm{u}^{2}}{2 !} \Delta^{2} \mathrm{y}_{-1}+\frac{\mathrm{u}\left(\mathrm{u}^{2}-1\right)}{3 !} \frac{\left(\Delta^{3} \mathrm{y}_{-2}+\Delta^{3} \mathrm{y}_{-1}\right)}{2}+\frac{\mathrm{u}\left(\mathrm{u}^{2}-1\right)}{4 !} \Delta^{4} \mathrm{y}_{2}+ \\
& \frac{\mathrm{u}\left(\mathrm{u}^{2}-1\right)\left(\mathrm{u}^{2}-2^{2}\right)}{5 !} \frac{\left(\Delta^{5} \mathrm{y}_{-3}+\Delta^{5} \mathrm{y}_{-2}\right)}{2}+\ldots \ldots
\end{aligned}
$$

\subsection{Bessel's Interpolation Formula}

For the derivation of Bessel's formula, taking the Mean of the Gauss's Forward formula and Third Gauss's formula thus we achieve the Bessel's Formula as

$$
\begin{aligned}
& \mathrm{y}(\mathrm{x})=\frac{\left(\mathrm{y}_{0}+\mathrm{y}_{1}\right)}{2}+\left(\mathrm{u}-\frac{1}{2}\right) \Delta \mathrm{y}_{0}+\frac{\mathrm{u}(\mathrm{u}-1)}{2 !} \frac{\left(\Delta^{2} \mathrm{y}_{-1}+\Delta^{2} \mathrm{y}_{0}\right)}{2}+\frac{\mathrm{u}\left(\mathrm{u}-\frac{1}{2}\right)(\mathrm{u}-1)}{3 !} \Delta^{3} \mathrm{y}_{-1}+\frac{\mathrm{u}\left(\mathrm{u}^{2}-1\right)(\mathrm{u}-2)}{4 !} \frac{\left(\Delta^{4} \mathrm{y}_{-2}+\Delta^{4} \mathrm{y}_{-1}\right)}{2} \\
& +\frac{\mathrm{u}\left(\mathrm{u}-\frac{1}{2}\right)\left(\mathrm{u}^{2}-1\right)(\mathrm{u}-2)}{5 !} \Delta^{5} \mathrm{y}_{-2}+\ldots \ldots \text { (5) }
\end{aligned}
$$

\subsection{Laplace-Everett's Formula}

This is a widely used interpolation formula as well as applies only even order differences, the formula has the form as

$$
\begin{aligned}
& \mathrm{y}(\mathrm{x})=\left[\mathrm{vy}_{0}+\frac{\left(\mathrm{v}^{2}-1\right) \mathrm{v}}{3 !} \Delta^{2} \mathrm{y}_{-1}+\frac{\left(\mathrm{v}^{2}-1\right) \mathrm{v}\left(\mathrm{v}^{2}-2^{2}\right)}{5 !} \Delta^{4} \mathrm{y}_{-2}+\cdots\right]+\quad\left[\mathrm{uy}_{1}+\frac{\left(\mathrm{u}^{2}-1\right) \mathrm{u}}{3 !} \Delta^{2} \mathrm{y}_{0}+\right. \\
& \left.\frac{\mathrm{u}\left(\mathrm{u}^{2}-1\right)\left(\mathrm{u}^{2}-2^{2}\right)}{5 !} \Delta^{4} \mathrm{y}_{-1}+\cdots\right] \ldots \ldots \text { (6) }
\end{aligned}
$$


Applied Mathematics and Sciences: An International Journal (MathSJ), Vol. 6, No. 2/3, September 2019

\subsection{New Interpolation Method}

To derive the new method first we need to derive the Gauss's Third formula which is derived by advancing the subscript of Gauss's backward formula by one unit and replacing $\mathrm{u}$ by $\mathrm{u}-1$. Then we get

$$
\begin{aligned}
& \mathrm{y}(\mathrm{x})=\mathrm{y}_{1}+(\mathrm{u}-1) \Delta \mathrm{y}_{0}+\frac{\mathrm{u}(\mathrm{u}-1)}{2 !} \Delta^{2} \mathrm{y}_{0}+\frac{\mathrm{u}(\mathrm{u}-1)(\mathrm{u}-2)}{3 !} \Delta^{3} \mathrm{y}_{-1}+\frac{\mathrm{u}\left(\mathrm{u}^{2}-1\right)(\mathrm{u}-2)}{4 !} \Delta^{4} \mathrm{y}_{-1}+ \\
& \frac{\mathrm{u}\left(\mathrm{u}^{2}-1\right)(\mathrm{u}-2)(\mathrm{u}-3)}{5 !} \Delta^{5} \mathrm{y}_{\cdot 2}+\ldots \ldots
\end{aligned}
$$

Now, we retreat the subscripts of Gauss's Forward formula in equation (1) by one unit and replacing $\mathrm{u}$ by $\mathrm{u}+1$ then we get

$$
\begin{aligned}
& \mathrm{y}(\mathrm{x})=\mathrm{y}_{-1}+(\mathrm{u}+1) \Delta \mathrm{y}_{-1}+\frac{\mathrm{u}(\mathrm{u}+1)}{2 !} \Delta^{2} \mathrm{y}_{-2}+\frac{\mathrm{u}(\mathrm{u}+1)(\mathrm{u}+2)}{3 !} \Delta^{3} \mathrm{y}_{-2}+\frac{\mathrm{u}(\mathrm{u}+1)\left(\mathrm{u}^{2}-2^{2}\right)}{4 !} \Delta^{4} \mathrm{y}_{-3}+ \\
& \frac{\mathrm{u}\left(\mathrm{u}^{2}-1\right)(\mathrm{u}+2)(\mathrm{u}+3)}{5 !} \Delta^{5} \mathrm{y}_{-3}+\ldots \ldots
\end{aligned}
$$

Therefore, taking the mean of the above two equation (7) and (8) i.e. adding (7) and (8) then dividing by 2 then we get a new method as

$$
\begin{aligned}
& \mathrm{y}(\mathrm{x})=\frac{\left(\mathrm{y}_{1}+\mathrm{y}_{-1}\right)}{2}+\mathrm{u}\left[\frac{\Delta \mathrm{y}_{-1}+\Delta \mathrm{y}_{0}}{2}\right] \\
& \frac{\mathrm{u}}{3 !}\left[\frac{\left(\mathrm{u}^{2}+3 \mathrm{u}+2\right) \Delta^{3} \mathrm{y}_{-2}+\left(\mathrm{u}^{2}-3 \mathrm{u}+2\right) \Delta^{3} \mathrm{y}_{-1}}{2}\right] \\
& \frac{\left(\mathrm{u}^{2}-1\right) \mathrm{u}}{5 !}\left[\frac{\left(\mathrm{u}^{2}+5 \mathrm{u}+6\right) \Delta^{5} \mathrm{y}_{-3}+\left(\mathrm{u}^{2}-5 \mathrm{u}+6\right) \Delta^{5} \mathrm{y}_{-2}}{2}\right]+\ldots \ldots
\end{aligned}
$$

This equation (9) is the required New Method of central difference interpolation.

\section{EXPERIMENTS}

Here, we have tested our proposed model by comparison test among all the formulas by some examples

Problem 1: In the given table, estimate the value of $y=\log _{10} 337.5$ by using (i) Gauss's forward interpolation formula (ii) Gauss's backward interpolation formula (iii) Stirling's formula (iv) Bessel's formula (v) Laplace Everett's formula and (vi) New proposed method.

\begin{tabular}{|c|l|l|l|l|l|l|}
\hline $\mathrm{x}$ & 310 & 320 & 330 & 340 & 350 & 360 \\
\hline $\mathrm{y}=\log _{10} \mathrm{x}$ & 2.4913617 & 2.5051500 & 2.5185139 & 2.5314789 & 2.544068 & 2.5563025 \\
\hline
\end{tabular}

Solution: Here $\mathrm{h}=10$, since we shall find $\mathrm{y}=\log _{10} 337.5$. Let us take 330 as the origin. $\mathrm{u}==\mathbf{=}=0.75$

$$
\mathrm{u}=\frac{\mathrm{x}-\mathrm{x}_{\mathrm{0}}}{\mathrm{h}}=\frac{337.5-330}{10}=0.75 \text { and } \mathrm{v}=1-\mathrm{u}=0.25
$$

Now we shall make the following difference table: 
Applied Mathematics and Sciences: An International Journal (MathSJ), Vol. 6, No. 2/3, September 2019

Table 1: Difference table for problem 1.

\begin{tabular}{|c|c|c|c|c|c|c|}
\hline $\mathrm{x}$ & $\mathbf{u}$ & $y$ & $\Delta \mathrm{y}$ & $\Delta^{2} y$ & $\Delta^{3} y$ & $\Delta^{4} y$ \\
\hline 310 & -2 & 2.4913617 & 0.0137883 & -0.0004244 & & \\
\hline 320 & -1 & 2.5051500 & 0.0133639 & & 0.0000255 & \\
\hline 330 & 0 & 2.5185139 & 0.012965 & -0.0003989 & & -0.0000025 \\
\hline 340 & 1 & 2.5314789 & $\begin{array}{l}0.0125891 \\
0.0122345\end{array}$ & -0.0003759 & 0.000023 & -0.0000017 \\
\hline 350 & 2 & 2.544068 & & -0.0003546 & 0.0000215 & \\
\hline 360 & 3 & 2.5563025 & & & & \\
\hline
\end{tabular}

(i) By Gauss's forward interpolation formula: we have,

$$
\begin{aligned}
& y(x)=y_{0}+u \Delta y_{0}+\frac{u(u-1)}{2 !} \Delta^{2} y_{\cdot 1}+\frac{u\left(u^{2}-1\right)}{3 !} \Delta^{3} y_{\cdot 1}+\frac{u\left(u^{2}-1\right)(u-2)}{4 !} \Delta^{4} y_{\cdot 2}+\frac{u\left(u^{2}-1\right)\left(u^{2}-2^{2}\right)}{5 !} \Delta^{5} y_{-2}+\ldots \\
& \begin{array}{ll}
y(337.5) \\
2.5185139+0.75
\end{array} \\
& +\frac{0.75 \times(0.75-1)}{2 !} \times-0.0003989+\frac{(0.75+1) \times 0.75 \times(0.75-1)}{3 !} \times 0.000023+ \\
& \frac{(0.75-2)(0.75+1) \times 0.75 \times(0.75-1)}{4 !} \times 0.0000025
\end{aligned}
$$

$=2.625787496$

Hence, the value of $\log _{10} 337.5=2.625787496$.

(ii) By Gauss's backward interpolation formula: we have, $\mathrm{y}(\mathrm{x})$

$\mathrm{y}_{0}+\mathrm{u} \Delta \mathrm{y}_{-1}+\frac{(\mathrm{u}+1) \mathrm{u}}{2 !} \Delta^{2} \mathrm{y}_{-1}+\frac{(\mathrm{u}+1) \mathrm{u}(\mathrm{u}-1)}{3 !} \Delta^{3} \mathrm{y}_{-2}+\frac{(\mathrm{u}+2)(\mathrm{u}+1) \mathrm{u}(\mathrm{u}-1)}{4 !} \Delta^{4} \mathrm{y}_{-2}+$ $\frac{(u+2)(u+1) u(u-1)(u-2)}{5 !} \Delta^{5} y_{-3}+\ldots$

$y(337.5)=2.5185139+0.75 \times 0.133639+\frac{(0.75+1) \times 0.75}{2 !} \times-0.0003989+\frac{(0.75+1) \times 0.75 \times(0.75-1)}{3 !} \times$ $0.0000255+\frac{(0.75+2)(0.75+1) \times 0.75 \times(0.75-1)}{4 !} \times-0.0000025$

$=2.5185139+0.10022925-0.000261778125-0.00000139453125+0.00000009399414063$

$=2.618480071$

Consequently, the value of $\log _{10} 337.5=2.618480071$.

(iii) By Stirling's formula: we have,

$\mathrm{y}(\mathrm{x})$

$\mathrm{y}_{0}+\frac{\mathrm{u}\left(\Delta \mathrm{y}_{0}+\Delta \mathrm{y}_{-1}\right)}{2}+\frac{\mathrm{u}^{2}}{2} \times \Delta^{2} \mathrm{y}_{-1}+\frac{\left(\mathrm{u}^{2}-1\right) \mathrm{u}}{3 !} \times \frac{\Delta^{3} \mathrm{y}_{-1}+\Delta^{3} \mathrm{y}_{-2}}{2}+\frac{\left(\mathrm{u}^{2}-1\right) \mathrm{u}^{2}}{4 !} \times \Delta^{4} \mathrm{y}_{-2}+\frac{\mathrm{u}\left(\mathrm{u}^{2}-1\right)\left(\mathrm{u}^{2}-2^{2}\right)}{5 !} \times$ $\frac{\left(\Delta^{5} y_{-2}+\Delta^{5} y_{-3}\right)}{2}+\ldots$

$\mathrm{y}(337.5)=2.5185139+0.009732375-0.000112190625-0.000001326171875$

$=2.528132784$

Hence the value of $\log _{10} 337.5=2.528132784$. 
Applied Mathematics and Sciences: An International Journal (MathSJ), Vol. 6, No. 2/3, September 2019

(iv) By Bessel's formula: we have, $\mathrm{y}(\mathrm{x})$

$\mathrm{y}(\mathrm{x})$

$=\frac{\mathrm{y}_{0}+\mathrm{y}_{1}}{2}+(\mathrm{u}-0.5) \times \Delta \mathrm{y}_{0}+\frac{(\mathrm{u}-1) \mathrm{u}}{2 !} \times \frac{\Delta^{2} \mathrm{y}_{-1}+\Delta^{2} \mathrm{y}_{0}}{2}+\frac{(\mathrm{u}-0.5) \mathrm{u}(\mathrm{u}-1)}{3 !} \times \Delta^{3} \mathrm{y}_{-1}+\frac{(\mathrm{u}+1) \mathrm{u}(\mathrm{u}-1)(\mathrm{u}-2)}{4 !} \times$ $\frac{\Delta^{4} \mathrm{y}_{-1}+\Delta^{4} \mathrm{y}_{-2}}{2}+\ldots$

$\mathrm{y}(337.5)=2.5249964+0.00324125+0.00003631875-0.0000001796875-0.00000003588867188$ $=2.557445003$

So, the value of $\log _{10} 337.5=2.557445003$.

(v) By Laplace-Everett's formula: we have,

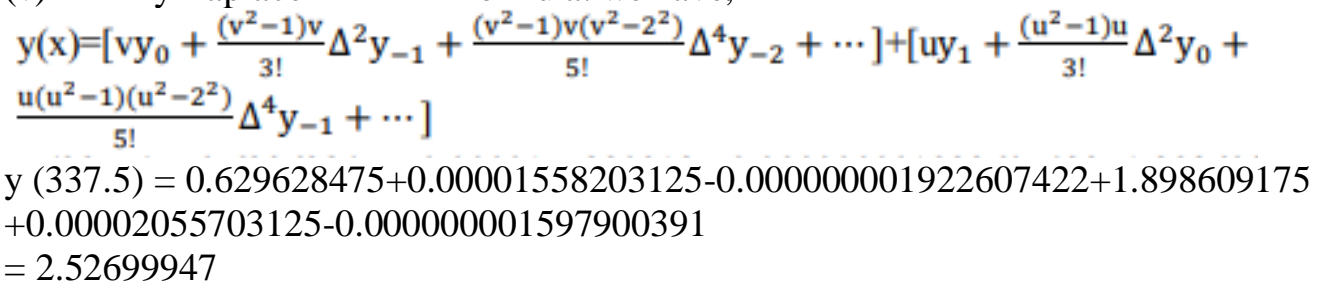

Therefore, the value of $\log _{10} 337.5=2.52699947$.

(vi) New proposed method: we have, $\mathrm{y}(\mathrm{x})$

$$
\begin{aligned}
& \mathrm{y}(\mathrm{x}) \\
& =\frac{\mathrm{y}_{1}+\mathrm{y}_{-1}}{2}+\frac{\mathrm{u}}{2} \times\left[\Delta \mathrm{y}_{0}+\Delta \mathrm{y}_{-1}\right]+\frac{\left(\Delta \mathrm{y}_{-1}-\Delta \mathrm{y}_{0}\right)}{2}+\frac{\mathrm{u}}{2 !}\left[\frac{(\mathrm{u}+1) \Delta^{2} \mathrm{y}_{-2}+(\mathrm{u}-1) \Delta^{2} \mathrm{y}_{0}}{2}\right]+ \\
& \frac{\mathrm{u}}{3 !}\left[\frac{\left(\mathrm{u}^{2}+3 \mathrm{u}+2\right) \Delta^{3} \mathrm{y}_{-2}+\left(\mathrm{u}^{2}-3 \mathrm{u}+2\right) \Delta^{3} \mathrm{y}_{-1}}{2}\right]+\frac{\left(\mathrm{u}^{2}-1\right) \mathrm{u}}{4 !}\left[\frac{(\mathrm{u}+2) \Delta^{4} \mathrm{y}_{-3}+(\mathrm{u}-2) \Delta^{4} \mathrm{y}_{-1}}{2}\right]+ \\
& \frac{\left(\mathrm{u}^{2}-1\right) \mathrm{u}}{5 !}\left[\frac{\left(\mathrm{u}^{2}+5 \mathrm{u}+6\right) \Delta^{5} \mathrm{y}_{-3}+\left(\mathrm{u}^{2}-5 \mathrm{u}+6\right) \Delta^{5} \mathrm{y}_{-2}}{2}\right]+\ldots \\
& \operatorname{Or}, \mathrm{y}(337.5)=\frac{2.5314789+0.5051500}{2}+\frac{0.75}{2}[0.0133639+0.012965]+\frac{0.0133639-0.012965}{2} \\
& \quad+\frac{0.75}{2 !}\left[\frac{(0.75+1) \times-0.0004244+(0.75-1) \times-0.0003759}{2}\right]+ \\
& \quad\left[\frac{\left(0.75^{2}+3 \times 0.75+2\right) \times 0.0000255+\left(\left(0.27^{2}-3 \times 0.75+2\right)\right) \times-0.0003989}{2}\right] \times \frac{0.75}{3 !}
\end{aligned}
$$

$\mathrm{y}(337.5)=2.51831445+0.0098733375+0.00019945-0.0001216359375-0.00000012109375$ $=2.52827358$

Hence, the value of $\log _{10} 337.5=2.52827358$.

Problem 2: The following table imparts the values of $f(x)=$ when $x=0.5437$,<smiles>C[AlH]</smiles>

calculate the value of the integral using (i) Gauss's forward interpolation formula, (ii) Gauss's backward interpolation formula, (iii) Stirling's formula, (iv) Bessel's formula, (v) LaplaceEverett's formula and (vi) New proposed method.

\begin{tabular}{|l|l|l|l|l|l|l|l|}
\hline $\mathrm{x}$ & 0.51 & 0.52 & 0.53 & 0.54 & 0.55 & 0.56 & 0.57 \\
\hline $\mathrm{y}$ & 0.5292437 & 0.5378987 & 0.5464641 & 0.5549392 & 0.563323 & 0.5716157 & 0.57981 \\
\hline
\end{tabular}

Solution: Here $\mathrm{h}=0.1$ and $\mathrm{x}=0.5437$. Let us take 0.54 as the origin. Thus, we can evaluate the value of $y$ for 
Applied Mathematics and Sciences: An International Journal (MathSJ), Vol. 6, No. 2/3, September 2019

$$
\mathrm{u}=\frac{\mathrm{x}-\mathrm{x}_{0}}{\mathrm{~h}}=\frac{0.5437-0.54}{0.1}=0.037 \text { and } \mathrm{v}=1-\mathrm{u}=1-0.037=0.963 \text {. }
$$

Now we will form the following central difference table:

Table 2. Difference table for problem 2.

\begin{tabular}{|c|c|c|c|c|c|l|l|}
\hline $\mathrm{x}$ & & $\mathrm{Y}$ & $\Delta \mathrm{y}$ & $\Delta^{2} \mathrm{y}$ & $\Delta^{3} \mathrm{y}$ & \multicolumn{1}{c|}{$\Delta^{4} \mathrm{y}$} & \multicolumn{1}{c|}{$\Delta^{5} \mathrm{y}$} \\
\hline 0.51 & -3 & 0.5292437 & 0.008655 & & & & \\
0.52 & -2 & 0.5378987 & & -0.0000896 & & & \\
0.53 & -1 & 0.5464641 & 0.008565 & & -0.0000007 & & \\
0.54 & 0 & 0.5549392 & 0.008475 & -0.0000903 & & 0.0 & 0.0 \\
0.55 & 1 & 0.5633233 & 0.0083841 & -0.000091 & -0.0000007 & 0.0 & 0.000000 \\
0.56 & 2 & 0.5716157 & 0.0082924 & -0.0000917 & -0.0000007 & 0.000000 & 1 \\
0.57 & 3 & 0.5798158 & 0.008200 & -0.0000923 & -0.0000006 & 1 & \\
& & & & & & & \\
\end{tabular}

(i) By Gauss's forward interpolation formula: we have,

$\mathrm{y}(0.5437)=0.5549392+0.0003102117+0.0000016212105+0.00000000431075715+$ 0.000000000123122337

$=0.5552510373$

Hence, the value of $\mathrm{y}(0.5437)=0.5552510373$.

(ii) By Gauss's backward interpolation formula: we have,

$\mathrm{y}(0.5437)=0.5549392+0.0003135787+0.0000017457895+0.00000000431075715$ $=0.5552510372$

Hence, the value of $\mathrm{y}(0.5437)=0.5552510372$.

(iii) By Stirling's formula: we have,

$\mathrm{y}(0.5437)=0.5549392-0.0000016835-0.0000000622895=0.00000000431075715$ $=0.555251062$

Hence, the value of $\mathrm{y}(0.5437)=0.555251062$.

(iv) By Bessel's formula: we have,

$\mathrm{y}(0.5437)=0.55913125-0.0038818383+0.0000106468425-0.000000006395141667$

$=0.5552600523$

$+0.0000000001511074337$

Hence, the value of $\mathrm{y}(0.5437)=0.5552600523$. 
Applied Mathematics and Sciences: An International Journal (MathSJ), Vol. 6, No. 2/3, September 2019

(v) By Laplace-Everett's formula: we have,

$y(0.5437)=0.5344064496+0.000001060812071+0.0208429621+0.0000005647091867$

$+0.00000000006154009195$

$=0.5552510373$

Hence, the value of $\mathrm{y}(0.5437)=0.5552510373$.

(vi) New proposed method: we have, $\mathrm{y}(\mathrm{x})$

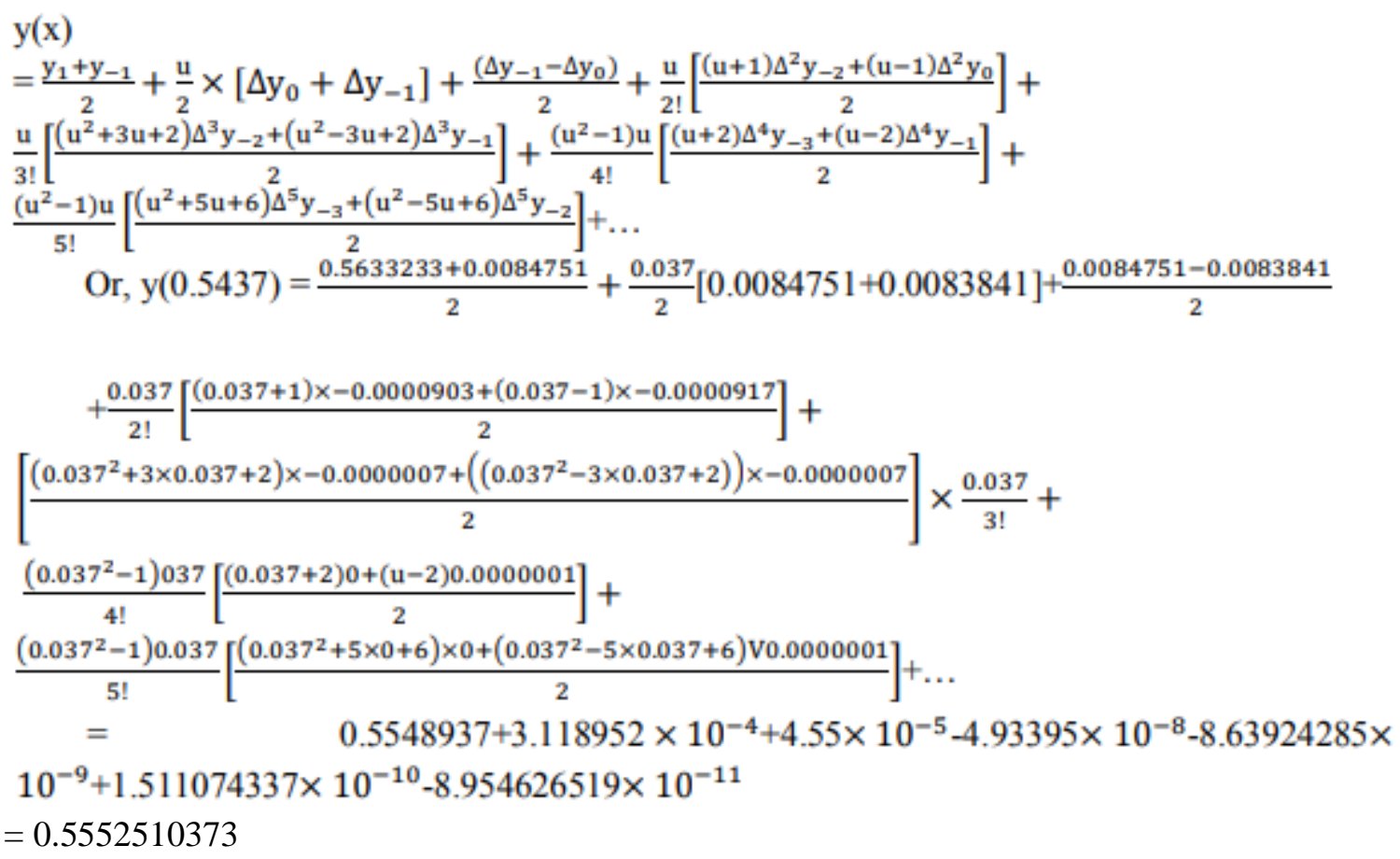

Hence, the value of $\log _{10} 337.5=0.5552510373$.

Problem 3: From the following data, find out the value of $\sin 45^{\circ}$ by exerting (i) Gauss's forward interpolation formula, (ii)Gauss's backward interpolation formula, (iii) Stirling's formula, (iv) Bessel's formula, (v) Laplace-Everett's formula and (vi) New proposed method.

\begin{tabular}{|l|r|r|r|r|r|}
\hline $\mathrm{X}$ & $20^{\circ} 30^{\circ}$ & $40^{\circ}$ & $50^{\circ}$ & $60^{\circ}$ & $70^{\circ}$ \\
\hline $\sin \mathrm{x}^{\circ}$ & 0.342020 .5 & 0.64278 & 0.76604 & 0.86602 & 0.93969 \\
\hline
\end{tabular}

Solution: Here $\mathrm{h}=10$. Let us take 50 as the origin. We have,

$$
\mathrm{u}=\frac{\mathrm{x}-\mathrm{x}_{0}}{\mathrm{~h}}=\frac{45-\overline{50}=-0.5}{10}
$$

and $\mathrm{v}=1-\mathrm{u}=1.5$

Now we will build the following central difference table: 
Applied Mathematics and Sciences: An International Journal (MathSJ), Vol. 6, No. 2/3, September 2019

Table 3. Difference table for problem 3.

\begin{tabular}{|c|ll|l|l|l|l|l|}
\hline $\mathrm{x}^{\circ}$ & \multicolumn{3}{|c|}{$\begin{array}{l}\text { y }=\mathrm{s} \\
\text { in } \mathrm{x}^{\circ}\end{array}$} & & & & \\
\hline 20 & -3 & 0.34202 & 0.15798 & & & \\
30 & -2 & 0.5 & 0.14278 & 0.0152 & -0.00432 & & \\
40 & -1 & 0.64278 & 0.12326 & -0.01952 & -0.00376 & 0.00056 & 0.00017 \\
50 & 0 & 0.76604 & 0.09998 & -0.02328 & -0.00303 & & \\
60 & 1 & 0.86602 & 0.07367 & -0.02631 & & & \\
70 & 2 & 0.93969 & & & & & \\
\hline
\end{tabular}

(i) By Gauss's forward interpolation formula: we have,

$\mathrm{y}\left(45^{\circ}\right)=0.76604-0.0499-0.00873-0.00012625+0.00001140625$

$=0.7072951563$

$\mathrm{y}\left(45^{\circ}\right)=0.7072951563$

(ii) By Gauss's backward interpolation formula: we have,

$\mathrm{y}\left(45^{\circ}\right)=0.76604-0.06163+0.00291-0.000235+0.000017109375-0.0000019921875$

$=0.7071001172$

$\mathrm{y}\left(45^{\circ}\right)=0.7071001172$

(iii) By Stirling's formula: we have,

$\mathrm{y}\left(45^{\circ}\right)=0.76604-005581-0.00291-0.0002121875-0.000005703125$

$=0.7071021094$

$\mathrm{y}\left(45^{\circ}\right)=0.7071021094$

(iv) Bessel's formula: we have,

$\mathrm{y}\left(45^{\circ}\right)=0.816035-0.09999-0.009298125+0.00038375$

$=0.707130625$

$\mathrm{y}\left(45^{\circ}\right)=0.707130625$.

(v) By Laplace-Everett's formula: we have,

$\mathrm{y}\left(45^{\circ}\right)=1.14906-0.0109125-0.000019960937-0.43301-0.001644375$

$=0.7034731641$

$\mathrm{y}\left(45^{\circ}\right)=0.7034731641$

(vi) New proposed method: we have,

$\mathrm{y}\left(45^{\circ}\right)=0.7544-0.05581+0.01164-0.003713125+0.0005909375$

$=0.7071078125$

$\mathrm{y}\left(45^{\circ}\right)=0.7071078125$. 
Applied Mathematics and Sciences: An International Journal (MathSJ), Vol. 6, No. 2/3, September 2019

Problem 4: From the following table, find $\mathrm{e}^{0.644}$ by applying (i) Gauss's forward interpolation formula, (ii) Gauss's backward interpolation formula, (iii) Stirling's formula, (iv) Bessel's formula, (v) Laplace Everett's formula and (vi) New proposed method.

\begin{tabular}{|l|r|r|r|r|r|r|r|}
\hline $\mathrm{x}$ & 0.61 & 0.62 & 0.63 & 0.64 & 0.65 & 0.66 & 0.67 \\
\hline $\mathrm{y}$ & 1.840431 & 1.858928 & 1.877610 & 1.896481 & 1.915541 & 1.934792 & 1.954237 \\
\hline
\end{tabular}

Solution: Here we have $\mathrm{x}=0.644, \mathrm{x}_{0}=0.64$ and since $\mathrm{h}=0.01$ then we have,

$$
\mathrm{u}=\frac{\mathrm{x}-\mathrm{x}_{0}}{\mathrm{~h}}=\frac{0.644-0.64}{0.01}=0.4 \text { and } \mathrm{v}=1-\mathrm{u}=1-0.4=0.6 \text {. }
$$

Now we will construct the following central difference table:

Table 4. Difference table for problem 4.

\begin{tabular}{|c|c|c|c|c|c|c|}
\hline $\mathrm{X}$ & $\mathrm{u}$ & $\mathrm{y}=\mathrm{e}^{\mathrm{x}}$ & \multicolumn{1}{|c|}{$\Delta y$} & $\Delta^{2} y$ & $\Delta^{3} y$ & $\Delta^{4} y$ \\
\hline 0.61 & -3 & 1.840431 & 0.018497 & & & \\
0.62 & -2 & 1.858928 & & 0.000185 & 0.000004 & -0.000004 \\
0.63 & -1 & 1.877610 & 0.018871 & 0.000189 & 0.0 & 0.000002 \\
0.64 & 0 & 1.896481 & 0.01906 & 0.000189 & 0.000002 & \\
0.65 & 1 & 1.915541 & 0.000191 & & 0.000001 \\
0.66 & 2 & 1.934792 & 0.019251 & & & \\
& & & 0.019445 & 0.000194 & & \\
0.67 & 3 & 1.954237 & & & & \\
\hline
\end{tabular}

(i) By Gauss's forward interpolation formula: we have,

$\mathrm{y}(0.644)=1.896481+0.007624-0.00002268-0.000000112+0.0000000448$

$=1.904082253$

The value of $\mathrm{e}^{0.644}=1.904082253$.

(ii) By Gauss's backward interpolation formula: we have,

$\mathrm{y}(0.644)=1.896481+0.0075484+0.00005292+0-0.0000000672$

$=1.904082253$

The value of $\mathrm{e}^{0.644}=1.904082253$.

(iii) By Stirling's formula: we have,

$\mathrm{y}(0.644)=1.896481+0.0075862+0.00001512-0.000000056-0.0000000112$

$=1.904082253$

The value of $\mathrm{e}^{0.644}=1.904082253$.

(iv) By Bessel's formula: we have, 
Applied Mathematics and Sciences: An International Journal (MathSJ), Vol. 6, No. 2/3, September 2019

$\mathrm{y}(0.644)=1.906011-0.001906-0.0000228+0.00000008+0.000000056$

$=1.904082336$

The value of $\mathrm{e}^{0.644}=1.904082336$.

(v) By Laplace-Everett's formula: we have,

$\mathrm{y}(0.644)=1.1378886-0.000012096+0.000000023296+0.7662164-0.000010696$

$+0.000000005152=1.904082236$

The value of $\mathrm{e}^{0.644}=1.904082236$

(vi) New proposed method: We have,

$\mathrm{y}(0.644)=1.8965755+0.0075862-0.0000945+0.000015+0.000000064+0.0000000784$

$=1.904082342$.

The value of $\mathrm{e}^{0.644}=1.904082342$.

\section{RESUlTS AND DisCUSSION}

Here we have discussed the results of different problems for different formulas on the above comparing with other formulas. As a results, we get the following table,

\begin{tabular}{|c|c|c|c|c|c|c|c|}
\hline \begin{tabular}{|l|} 
Proble $m$ \\
no
\end{tabular} & $\begin{array}{l}\text { Gauss's } \\
\text { forward }\end{array}$ & Error & $\begin{array}{l}\text { Gauss's } \\
\text { backward }\end{array}$ & Error & Stirling's & Error & Exact value \\
\hline & $\begin{array}{l}2.62578749 \\
6\end{array}$ & $\begin{array}{l}9.751 \\
\%\end{array}$ & $\begin{array}{l}2.61848007 \\
1\end{array}$ & $\begin{array}{l}9.0207 \\
\%\end{array}$ & $\begin{array}{l}2.52813278 \\
4\end{array}$ & $\begin{array}{l}0.0141 \\
\%\end{array}$ & $\begin{array}{l}2.52827377 \\
7\end{array}$ \\
\hline & $\begin{array}{l}0.55525103 \\
73\end{array}$ & $0.29 \%$ & $\begin{array}{l}0.55525103 \\
72\end{array}$ & $0.29 \%$ & $\begin{array}{l}0.55493745 \\
8\end{array}$ & $\begin{array}{l}0.31146 \\
\%\end{array}$ & $\begin{array}{l}0.55805202 \\
1\end{array}$ \\
\hline & $\begin{array}{l}0.70729515 \\
63\end{array}$ & $\begin{array}{l}0.018 \\
\%\end{array}$ & $\begin{array}{l}0.70710011 \\
72\end{array}$ & $\begin{array}{l}0.0007 \\
\%\end{array}$ & $\begin{array}{l}0.70710210 \\
94\end{array}$ & $\begin{array}{l}0.00047 \\
\%\end{array}$ & $\begin{array}{l}0.70710678 \\
12\end{array}$ \\
\hline 4 & $\begin{array}{c}1.90408225 \\
3\end{array}$ & $\begin{array}{l}2.6 \\
\%\end{array}$ & $\begin{array}{c}1.90408225 \\
3\end{array}$ & $\begin{array}{l}2.6 \\
\%\end{array}$ & $\begin{array}{c}1.90408225 \\
3\end{array}$ & $\begin{array}{c}2.6 \\
\%\end{array}$ & $\begin{array}{c}1.90408199 \\
5\end{array}$ \\
\hline $\begin{array}{c}\text { Proble } \mathrm{m} \\
\text { No }\end{array}$ & Bessel's & Error & Everett's & Error & New method & Error & Exact value \\
\hline 1 & $\begin{array}{c}2.55744500 \\
3\end{array}$ & $\begin{array}{c}2.917 \\
\%\end{array}$ & 2.52699947 & $\begin{array}{c}0.1274 \\
\%\end{array}$ & 2.52827358 & $\begin{array}{c}0.00001 \\
\%\end{array}$ & $\begin{array}{c}2.52827377 \\
7\end{array}$ \\
\hline 2 & $\begin{array}{c}0.55525106 \\
2\end{array}$ & $0.29 \%$ & $\begin{array}{c}0.55525103 \\
73\end{array}$ & $0.29 \%$ & $\begin{array}{c}0.55525103 \\
73\end{array}$ & $\begin{array}{c}0.28009 \\
\%\end{array}$ & $\begin{array}{c}0.55805202 \\
1\end{array}$ \\
\hline 3 & $\begin{array}{c}0.70713062 \\
5\end{array}$ & $\begin{array}{c}0.002 \\
\%\end{array}$ & $\begin{array}{c}0.70347316 \\
41\end{array}$ & $\begin{array}{c}0.3634 \\
\%\end{array}$ & $\begin{array}{c}0.70710781 \\
25\end{array}$ & $\begin{array}{c}0.0002 \\
\%\end{array}$ & $\begin{array}{c}0.70710678 \\
12\end{array}$ \\
\hline 4 & $\begin{array}{c}1.90408233 \\
6\end{array}$ & $\begin{array}{c}3.5 \\
\%\end{array}$ & $\begin{array}{c}1.90408223 \\
6\end{array}$ & $\begin{array}{c}2.5 \\
\%\end{array}$ & $\begin{array}{c}1.90408234 \\
2\end{array}$ & $\begin{array}{c}2.4 \\
\%\end{array}$ & $\begin{array}{c}1.90408199 \\
5\end{array}$ \\
\hline
\end{tabular}

From the upon table, we can easily perceive that the values of the propound method are very close to the exact value for the sake of the various problems. Therefore, the new method is formally useful as well as gives lesser error values by virtue of graphical comparison between remaining methods. 

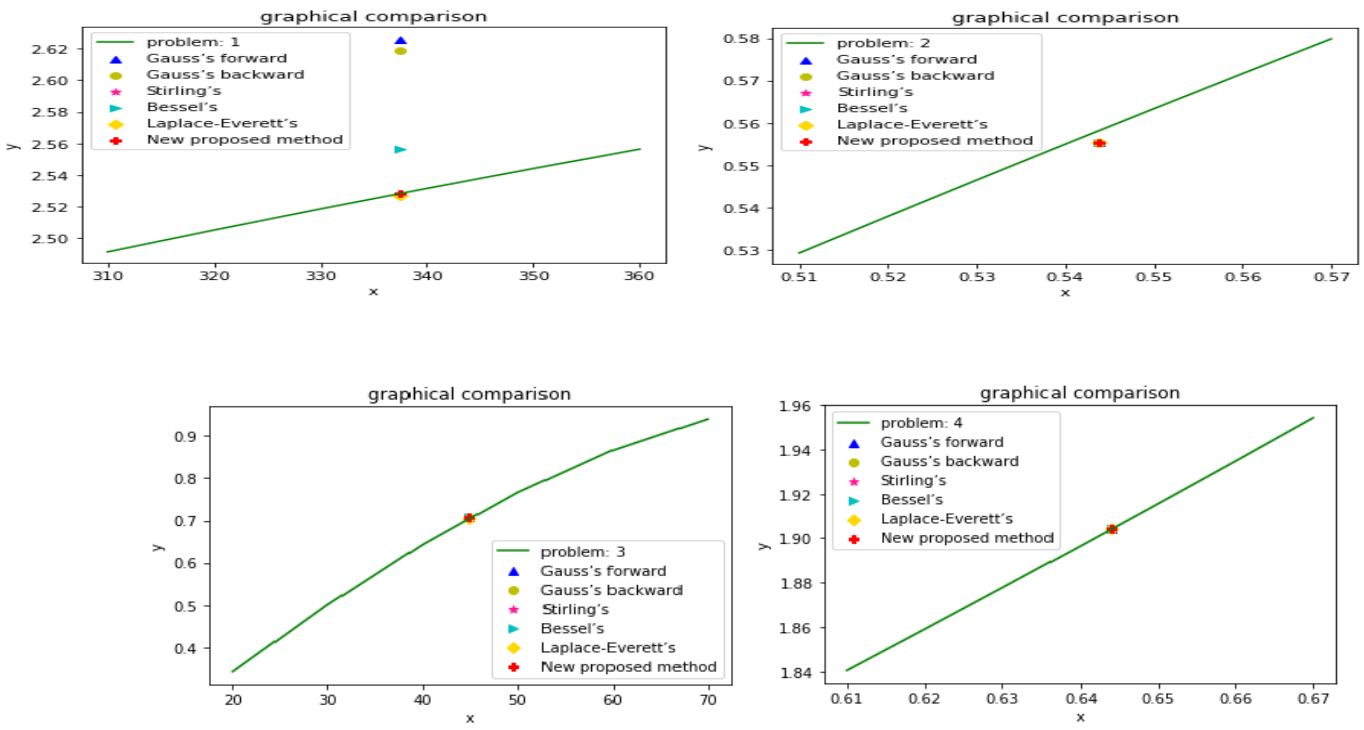

Figure 1. Graphical comparison.

From the fundamental observation in our paper, we have shown that:

* The propound method imparts a smaller value of the error, a higher rate of convergence compared to the other existing formulas.

* We have compared our new method graphically to demonstrate the most proper method with the help of the given examples.

\section{CONCLUSION}

After the whole study and work on our paper, we are able to propose the new method with regard to exactness, even the proposed method delivers better accuracy among the mentioned formulas for which our new method is the best in central difference interpolation. According to these methods examined, it was found that the value of the propound method is very nearly to the exact value of the several problems. That's why we have agreed that this new method is extremely effective as well as perhaps correct to provide a good accuracy rather than the other existing central difference interpolation formulas owing to calculating functional values between the given data.

\section{REFERENCES}

[1] Baak, M., Gadatsch, S., Harrington, R., \& Verkerke, W. (2015). Interpolation between multidimensional histograms using a new non-linear moment morphing method. Nuclear Instruments and Methods in Physics Research Section A: Accelerators, Spectrometers, Detectors and Associated Equipment, 771, 39-48.

[2] Bhunia, Gouri Sankar, Pravat Kumar Shit, and Ramkrishna Maiti. "Comparison of GIS- based interpolation methods for spatial distribution of soil organic carbon (SOC)." Journal of the Saudi Society of Agricultural Sciences 17.2 (2018): 114-126.

[3] Gentilucci, Matteo, Carlo Bisci, Peter Burt, Massimilano Fazzini, and Carmela Vaccaro. "Interpolation of Rainfall Through Polynomial Regression in the Marche Region (Central Italy)." In The Annual International Conference on Geographic Information Science, pp. 55-73. Springer, Cham, 2018. 
Applied Mathematics and Sciences: An International Journal (MathSJ), Vol. 6, No. 2/3, September 2019

[4] Zhang, JiHong, JunSheng Zheng, and QinJiao Gao. "Numerical solution of the Degasperis-Procesi equation by the cubic B-spline quasi-interpolation method." Applied Mathematics and Computation 324 (2018): 218-227.

[5] Su, Y., Zhang, Q., Xu, X., Gao, Z. and Wu, S., 2018. Interpolation bias for the inverse compositional Gauss-Newton algorithm in digital image correlation. Optics and Lasers in Engineering, 100, pp.267-278.

[6] Dehghan, Mehdi, and Mostafa Abbaszadeh. "The solution of nonlinear Green-Naghdi equation arising in water sciences via a meshless method which combines moving kriging interpolation shape functions with the weighted essentially non-oscillatory method." Communications in Nonlinear Science and Numerical Simulation 68 (2019): 220-239.

[7] Li, J., Heap, A. D., Potter, A., \& Daniell, J. J. (2011). Application of machine learning methods to spatial interpolation of environmental variables. Environmental Modelling \& Software, 26(12), 1647-1659.

[8] Bui, T. Q., Khosravifard, A., Zhang, C., Hematiyan, M. R., \& Golub, M. V. (2013). Dynamic analysis of sandwich beams with functionally graded core using a truly meshfree radial point interpolation method. Engineering structures, 47, 90-104.

[9] Ghehsareh, H. R., Zaghian, A., \& Zabetzadeh, S. M. (2018). The use of local radial point interpolation method for solving two-dimensional linear fractional cable equation. Neural Computing and Applications, 29(10), 745-754.

[10] Jung, K. H. (2018). A survey of interpolation-based reversible data hiding methods. Multimedia Tools and Applications, 77(7), 7795-7810.

[11] D. C. Fraser (1927). Newton and interpolation. In Isaac Newton 1642-1727: A Memorial Volume, W. J. Greenstreet, Ed. London, U.K.: Bell, 45-69.

[12] J. D. Everett (1900). On a central-difference interpolation formula. Rep. Br. Assoc. Adv. Sci., 70, 648-650. J. D. Everett (1901). On a new interpolation formula. J. Inst. Actuar. 35, 452-458.

[13] R. C. Gupta (1969). Second order interpolation in Indian mathematics up to the fifteenth century. Ind. J. Hist. Sci., 4(1-2), 86-98.

[14] Omar A. AL-Sammarraie; Mohammed Ali Bashir ; Omdurman Islamic University ; Neelain University.International Journal of Scientific and Research Publications, Volume 5, Issue 3, March 2015 ISSN 2250-3153 www.ijsrp.org Generalization of Newton's Forward Interpolation Formula •

[15] W.F. Sheppard (1899). Central-difference formula. In Proceedings of the London Mathematical Society, 31, 449-488.

[16] H. H. Goldstine (1977). A History of Numerical Analysis from the 16th through the 19th Century. Berlin, Germany: Springer-Verlag.

[17] D. C. Fraser (1927). Newton's Interpolation Formulas. London, U.K.: C. \& E. Layton.

[18] F. B. Hildebrand (1974). Introduction to Numerical Analysis. New York: McGraw-Hill. 